\title{
Aprendizaje en el saber matemático en los docentes de las instituciones públicas de básica primaria del municipio Valledupar
}

\section{learning processes in the mathematical knowledge in the teachers of the public institutions of primary basics of the valledupar municipality.}

\section{Dr. Teovaldo García Romero ${ }^{1}$}

Dr. (c). Wilcar Damián Cifuentes Álvarez ${ }^{2}$

\section{Dr. Leonardo Enrique Martínez Arredondo ${ }^{3}$}

Universidad Popular del Cesar

\section{RESUMEN}

La presente investigación tiene como objetivo analizar el proceso de aprendizaje del saber matemático en los docentes de las instituciones

1 Dr. Teovaldo García Romero, Universidad Popular del Cesar; teovaldogarcia@unicesar.edu.co.iD.0000-00027233-4064

2 Dr. (c). Wilcar Damián Cifuentes Álvarez .

Universidad Popular del Cesar wilcarcifuentes@unicesar. edu.co. iD. 0000-0001-5565-6718

3 Dr. Leonardo Enrique Martínez Arredondo. Universidad Popular del Cesar leonardomartinez@unicesar. edu.co. iD. 0000-0002-9285-3320 públicas de básica primaria del Municipio Valledupar. Está fundamentada en las posturas teóricas de Maturana (2015), Echeverría (2013) Urquiza (2005), Flores (2000); entre otros, que permite una mirada distinta sobre el contexto educativo. Se desarrolla desde un enfoque positivista de tipo descriptivo, no experimental, transeccional, de campo. Para la población se seleccionaron 16 instituciones educativas, siendo la muestra 46 docentes con características homogéneas. Se aplicó la 
técnica de la encuesta y el cuestionario, siendo la confiabilidad de 0,85 altamente confiable. Los resultados evidencian la necesidad de generar nuevas formas de aprendizaje sobre el saber matemático que converjan con los intereses del estudiante para el logro de un aprendizaje significativo; por lo tanto, hoy requiere un compromiso efectivo que conduce a la interiorización de nuevas competencias para enfrentar el desafío de las exigencias actuales consecuentes a la transformación del sistema local, regional y nacional.

\section{PALABRAS CLAVE.}

Proceso de aprendizaje, saber matemático, educación básica.

\section{ABSTRAC}

The present research aims to establish the learning process of mathematical knowledge in teachers of public elementary schools of the Valledupar Municipality, it is based on the theoretical positions of Maturana (2015), Echeverría (2013) Urquiza (2005), Flores (2000); among others, that allows a different look at the educational context. It is developed from a positivist approach of a descriptive, non-experimental, transectional, field type. For the population, 16 educational institutions were selected, the sample being 46 teachers with homogeneous characteristics. The survey and questionnaire technique was applied, with a reliability of 0.85 being highly reliable. The results show the need to generate new ways of approaching mathematical knowledge that converge with the student's interests to achieve meaningful learning; Therefore, today it requires an effective commitment that leads to the internalization of new competences to face the challenge of current demands resulting from the transformation of the local, regional and national system.

\section{KEYWORDS}

Learning process, knowing mathematics, basic education.

\section{INTRODUCCIÓN}

Los avances a nivel global, han generado cambios impactando la historia, se viven momentos inciertos, causados por los grandes desafíos que se ocultaban en el futuro y que ya invaden los espacios predilectos del presente, los momentos actuales ha traído como consecuencia incertidumbres un tanto desconcertante, pero observables en un sistema educativo el cual se deslizan sobre una pista resbalosa y está sometida a dinámicas de transformación que exige actuar de una manera diferente.

Bajo esta mirada, la educación del siglo XXI exige la transformación del paradigma utilizado en el quehacer docente, donde los procesos de aprendizaje requieren una recontextualización, pues aun cuando los cambios se apoderan del sistema los resultados siguen jerarquizándose por encima de los procesos. No obstante, a nivel latinoamericano, la educación de básica primaria está enfrentadas a grandes desafíos en el área económica, social y política como consecuencias de la globalización; lo cual repercute en los procesos de aprendizaje en el saber matemático influenciados por las variables que convergen en la institución, de esta manera, los resultados esperados en el escenario académico de los estudiantes pueden ser fluctuantes dependiendo de las exigencias del entorno. 
Colombia no escapa de esta realidad, al respecto (Solaz, López y Gómez, 2011), afirman que los escenarios actuales de las instituciones públicas de básica primaria en Valledupar, por ser representativos de la ola de cambios, requieren de transformación; sin embargo, parecen estar alejadas de esta realidad, panorama que se observa por ausencia de preparación en los docentes para desafiar los retos, desmotivación para la ejecución de sus acciones, esquemas tradicionales en la enseñanza del saber matemático caracterizadas por diseños memorísticos que distan de la experiencia individual del sujeto, así como también curvas bajas de rendimiento académico; entre otras, lo cual impide obtener resultados óptimos en los procesos de aprendizaje.

Cabe destacar, que la mayor causa de los fracasos de los docentes en su rol de líder y formador en el contexto de básica primaria de las instituciones públicas de Valledupar, subyacen en el desconocimiento que tienen sobre la función del cerebro como órgano que permite procesar lo que se percibe para luego transformar y emitir una respuesta con una interpretación particular, seguido por la ausencia de competencias sobre los procedimientos que se llevan a cabo para instaurar nuevos esquemas mentales, lo cual dificulta la comprensión sobre como es el proceso de aprendizaje del saber matemático y cuáles son las etapas que se deben tener presentes para darle sentido y significado a lo aprendido.

Bajo el mismo orden, en la mayoría de los casos la percepción de las matemáticas versa sobre la interpretación "difícil de aprender" y los resultados de rendimiento académico de los estudiantes parecen confirmarlo, aun cuando hay vertientes pedagógicas que apuestan a mejorar el proceso. Siguiendo con la idea, Echeverría (2013) confirma que los procesos de aprendizaje son etapas necesarias para lograr el significado de lo que se aprende; al igual que el saber matemático, se va gestando gradualmente como competencia consciente e inconsciente del sujeto hasta lograr los resultados esperados.

Condición, que obliga al docente a la adquisición de competencias que refuercen su quehacer pedagógico orientando el logro de un aprendizaje de tercer orden o transformacional; de tal manera que a través de sus acciones provoque un cambio de conducta con efecto domino en el estudiante, donde la experiencia se conserve en el tiempo para ser trasladada a otros escenarios, abriendo las posibilidades de enfrentar los desafíos impuestos por el entorno con una perspectiva crítica utilizando lo aprendido para poder distinguir y jerarquizar lo útil.

Sobre el asunto, (Muniz y Borges, 2008), manifiestan que el aprendizaje de los contenidos matemáticos se hace significativo cuando el docente reconoce el cómo se procesa y cuáles son las etapas del mismo; lo cual, le permite utilizar estrategias cónsonas a las necesidades de los estudiantes para que puedan desglosar una idea, analizarla y comprenderla de forma sistemática hasta llegar a su profundidad; por tanto cada unidad de enseñanza tiene que ser preparada en función de la realidad, las edades y el nivel de formación de quien recibe la información.

Esta base teórica y sólida es fundamental para el entrenamiento profesional de un docente de básica primaria a fin de modificar su observador y trabajar constructivamente con cuatro campos fundamentales de la existencia humana el ser, hacer, conocer y convivir como medio de apoyo para desarrollar nuevas e importantes configuraciones que generen aprendizajes con significado. De ahí que, el complejo entorno de 
habituales de enseñanza aprendizaje dentro del salón de clases, resultan insuficientes para lograr los resultados que se necesita, por lo que los paradigmas de ayer ya no funcionan para resolver los problemas de hoy, implicando nuevos retos en función de la efectividad y bienestar dentro de la incertidumbre del presente.

En virtud de ello, todo aprendizaje constituye un proceso complejo, que finalmente se expresa en una modificación de la conducta y debe orientarse a acortar brechas que dificultan la enseñanza del saber, disminuyendo lo que Wolk (2007) llama la fisura entre lo que se sabe y lo que se desea saber, entre lo que se tiene y lo que se quiere, partiendo de nuevas propuestas orientadas por el docente hacia la autonomía del estudiante que conlleven a acciones distintas a las iniciales con resultados efectivos. Atendiendo a las consideraciones anteriores, el llamado de atención hacia los docentes se enfoca en reflexionar sobre el cómo y el para qué se aprende, en consecuencia, abandonar los viejos paradigmas para obtener resultados satisfactorios.

Una mirada distinta sobre el proceso de aprendizaje en el saber matemático, le otorga al docente la posibilidad de flexibilizar su estructura, fortalecer la seguridad en los procedimientos e ingresar nuevas estrategias que inviten al estudiante a mantener actitudes favorables para comprender, así como la confianza en los resultados obtenidos en pro del mejoramiento de un sistema educativo propio de la región, el estado y el país. De acuerdo, a lo anterior se plantea la siguiente interrogante ¿cómo el proceso de aprendizaje influye en el saber matemático?

En este sentido, surge la necesidad en el quehacer pedagógico de analizar el aprendizaje, estableciendo sus procesos y caracterizando las etapas del saber matemático para reforzar el quehacer pedagógico del docente comprometiéndolo a enfrentarse con la complejidad que se presenta en la comprensión del saber específico, haciendo consciente que su principal objetivo está en apoyar el desarrollo del estudiante como ser biopsicosocial fortaleciendo un pensamiento crítico, creativo, reflexivo y científico basado en fundamentos sólidos.

\section{A) CUERPO}

\section{Procesos de aprendizaje.}

La educación que demanda, hoy la sociedad obliga al docente a revisar su accionar frente a los procesos de aprendizaje; por tal razón, el profesional de la educación actual requiere de un comportamiento adaptado a las nuevas circunstancias, con pensamiento flexible, capacidad de surfear la ola y una mirada distinta que enfoque las competencias del saber especifico en un ángulo de $360^{\circ}$, para enfrentar los retos que acontecen cada día, que le permitan abrazar las circunstancias cambiantes del entorno y las condiciones de incertidumbre, Echeverría (2013).

Al ubicarse en este panorama Woolfolk (2006:33), afirma que el proceso de aprendizaje se relaciona directamente con acciones efectivas y afectivas. "Aprender no es solo tener información; constituyen un proceso complejo donde intervienen aspectos relacionantes como la experiencia, las emociones y el conocimiento para expandir la capacidad de acción efectiva, incrementando las competencias para poder operar en un determinado dominio que antes desconocía"; es decir, significa incorporar habilidades 
que hagan posible acceder a resultados $\mathrm{u}$ objetivos que antes estaban fuera del alcance; que finalmente se expresa en una modificación de la conducta. El gran desafío es aprender a aprender.

Por su parte Echeverría (2007), manifiesta que aprender a aprender implica apresurarse a la actitud de desaprender, (habilidad para soltar). Los modelos mentales, usados a través de años en ocasiones pueden convertirse en formas obsesivas de hacer docencia, por lo que actuar bajo una nueva filosofía del aprendizaje convierte cualquier encuentro de enseñanza en un momento enriquecedor, definiendo metas, prioridades y criterios de desempeño; por ello, que las competencias más visibles que se le solicitan a un docente es el actuar con influencia, empatía e intuición.

Bajo este paradigma, el autor propone el modelo O.S.A.R (observación, sistema, acción y resultado) tomando como punto de partida las experiencias del individuo que subyacen en un comportamiento que, a través del aprendizaje puede transformarse. Con la revisión de acciones, el observador establece una mirada crítica sobre la situación problemática se modifican conductas con nuevas acciones y se obtiene resultados diferentes. Cabe acotar, que en el proceso de aprendizaje no existe una realidad absoluta, cada docente se convierte en un observador distinto que interpreta el contexto según sus experiencias; cuando apertura su mente hacia nuevos esquemas de pensamiento, fácilmente acepta las diferencias de los estudiantes y busca las opciones para liderar con ellas, cuando algo se asume como realidad absoluta, es solo el producto de un mayor grado de consenso sobre la interpretación común que un gran número de observadores lo hace posible.
Cuando se toma como relevante lo anterior, el docente tiene la capacidad para construir significados que fortalezcan el saber matemático desde la acción, reflexión y análisis en conjunto, reflejando un lenguaje especifico coherente con códigos propios de la disciplina mientras se descubre en su uso otras reglas y convencionalismos. En virtud de lo anterior, se establece el proceso de aprendizaje en tres niveles:

- $\quad$ Aprendizaje de primer orden. Está constituido, por las experiencias vividas y la repetición de las mismas en el accionar diario; es decir, las acciones realizadas en el quehacer docente serian el producto de una observación e interpretación de lo externo, para llevarlos a la realidad de un salón de clase bien sea presencial o virtual; esta etapa implica, la organización cognitiva que comienza con la asimilación del aprendizaje definida por Piaget, como la repetición y generalización de una acción.

Al respecto Pólya (1989), expresa que el docente tiene una gran oportunidad para fomentar la curiosidad y creatividad de sus estudiantes, a través de las preguntas que verifiquen las experiencias previas planteándoles problemas y apoyando para posibles soluciones motivando e incentivando al desarrollo de un pensamiento independiente.

Al interpretar lo que se observa despejando las interrogantes, inconscientemente se descarta la información que no es de utilidad; magnificando algunos elementos se disminuyen otros, incluso, se añaden otros que no existían; haciendo énfasis en lo útil, el docente deja de lado la improvisación, apostando por una planificación con criterios de flexibilidad, atendiendo a la evolución de los hechos que se desarrollan en un determinado grupo. 
Es aquí, cuando la observación y los fenómenos interpretativos del docente y el estudiante producen cambios en laforma de interpretarloque sucede; es decir, si se modifican las perspectivas en la enseñanza del saber matemático, automáticamente cambia la interpretación de sus estudiantes; situación reconocida como un aprendizaje de primer orden, que dará lugar a la construcción de diferentes acciones y finalmente se transformará el resultado de las mismas; por tanto, lo que el docente ve, también lo verán sus estudiantes. Bajo este escenario, el proceso de aprendizaje coordinado por el docente, requiere de acciones diferentes en busca del logro de los objetivos propuestos, cuando se producen los resultados esperados se refuerza la efectividad y el comportamiento; cuando no coinciden con los objetivos se presenta una brecha de conocimiento.

\section{- Aprendizaje de segundo orden.} Está representado, por la modificación de las experiencias vividas en función de lograr resultados diferentes Echeverría (2007). Bajo la teoría piagetiana, esta etapa representa la acomodación donde se modifican los esquemas previos para hacer consciente nuevas experiencias y otorgarles un significado. En este nivel de aprendizaje, se requiere profundizar en las acciones, incorporando elementos nuevos en la ejecución de las tareas, lo cual permite tener otra perspectiva de la situación problémica y brindarle al estudiante la posibilidad de llegar al resultado desde distintas perspectivas, aquí se puede observar cómo se modifican paulatinamente las interpretaciones con referencia a la forma del cómo se aprende.

Por tanto, ordenar, clasificar, reunir o disociar, es fundamental para fomentar el pensamiento matemático, así pues, es necesario un ejercicio prolongado de acciones que conlleve a la movilización de las estructuras cognitivas básicas y superiores, esgrimiendo la construcción de un aprendizaje con representaciones concretas, comprensión del lenguaje respuesta social, así como fortalecer la interacción del docente y el estudiante coadyuvando en la edificación del saber matemático.

Considerando lo expuesto, el aprendizaje de segundo orden también recibe el nombre de segundo camino, donde el docente reconoce la posibilidad de no intervenir directamente en la acción, actuando a través de la facilitación del aprendizaje desde el casillero del estudiante; es decir, en el momento que se produzcan las acciones su intervención estará presta a la evaluación y reflexión sobre el mismo verificando el cambio de conducta.

En este orden de ideas, Chiva (2012) caracteriza el aprendizaje de segundo orden por la implementación de nuevas rutinas, procesos o propósitos que dejan atrás las acciones de los viejos paradigmas del docente, interpretando las creencias con un esquema distinto organizado y vigente que le otorga la posibilidad, de construir otras estrategias en función de percibir con otra mirada la información que el medio le provee, originando una forma diferente de aprender. En consecuencia, conlleva a los docentes de las instituciones básicas primaria, a desarrollar estrategias para lograr un aprendizaje en el saber matemático que conecten con la emocionalidad de los estudiantes en función de un desempeño eficiente a corto plazo, adaptándose incluso a situaciones inesperadas condicionantes del día a día. 
- Aprendizaje de tercer orden, transformacional. En esta etapa, el rol del docente como líder transformacional, es trascendental para el aprendizaje pues tiene el reto de integrar el área cognitiva, conductual, afectiva en un solo momento desde el momento de la planificación hasta sus acciones reflejadas en su discurso y en la incorporación, de nuevas estrategias con resultados efectivos, así como considerar la participación de todos los actores de la institución, sincronizando la practica con la reflexión en el aula y fuera de ella, ensayando estilos distintos de enseñanza aprendizaje adecuándose a las nuevas tendencias.

En este caso, para cimentar el aprendizaje del tercer orden se requiere ir más allá y revisar las creencias, valores, así como los modelos mentales que constituyen la conducta de los docentes para producir un cambio transformacional; estas circunstancias, vinculan el ser con el hacer y con el convivir para un desarrollo potencial e integral. Desde la perspectiva de Piaget, constituye la etapa de adaptación y considerando la postura de Vygotsky, se produce un andamiaje a través del proceso relacional, de tal manera que exista la apropiación gradual de un saber especifico con la orientación del experto.

Para tal efecto, se considera dos ideas importantes, la primera enfocada en el pensamiento (comprensión y producción de símbolos) y la segunda enmarcada en la función social (relaciones, apropiación de conocimientos); de tal manera, que las practicas realizadas en el salón, requieren de coherencia para edificar el saber matemático con significado sostenido en el tiempo, lo cual significa que el desarrollo cognitivo no tiene lugar de forma aislada, sino que transcurre junto al desarrollo del lenguaje, el desarrollo social e incluso el desarrollo físico. Echeverría (2013).

Para Roegiers. (2004), este nivel persigue la transformación del aprendizaje y la optimización del fluir relacional entre estudiantes y docentes, donde el discurso incluye un pensamiento estratégico relevante para lograr los objetivos propuestos. El propósito entonces, es crear condiciones que promuevan nuevas formas de hacer conocimiento, evitando el desvió de la concentración en normar los procesos o coartar la creatividad y libertad de los actores o en limitar la asunción de sus responsabilidades.

En este sentido, el énfasis del propósito del aprendizaje transformacional provee al docente de básica primaria, la capacidad de establecer bases sólidas para crear las estrategias de desarrollo necesarias, que converjan en la evolución de una institución que aprende. Según Mier (2004), un aprendizaje transformacional produce cambios con efectos significativos, en las experiencias subsiguientes del docente y estudiante ya que al cambiar la mirada el entorno también lo hace. Lo anterior. puede representarse en "el modelo del observador, sistema, acción y resultados”. Gráficamente lo presentamos de la siguiente manera: 


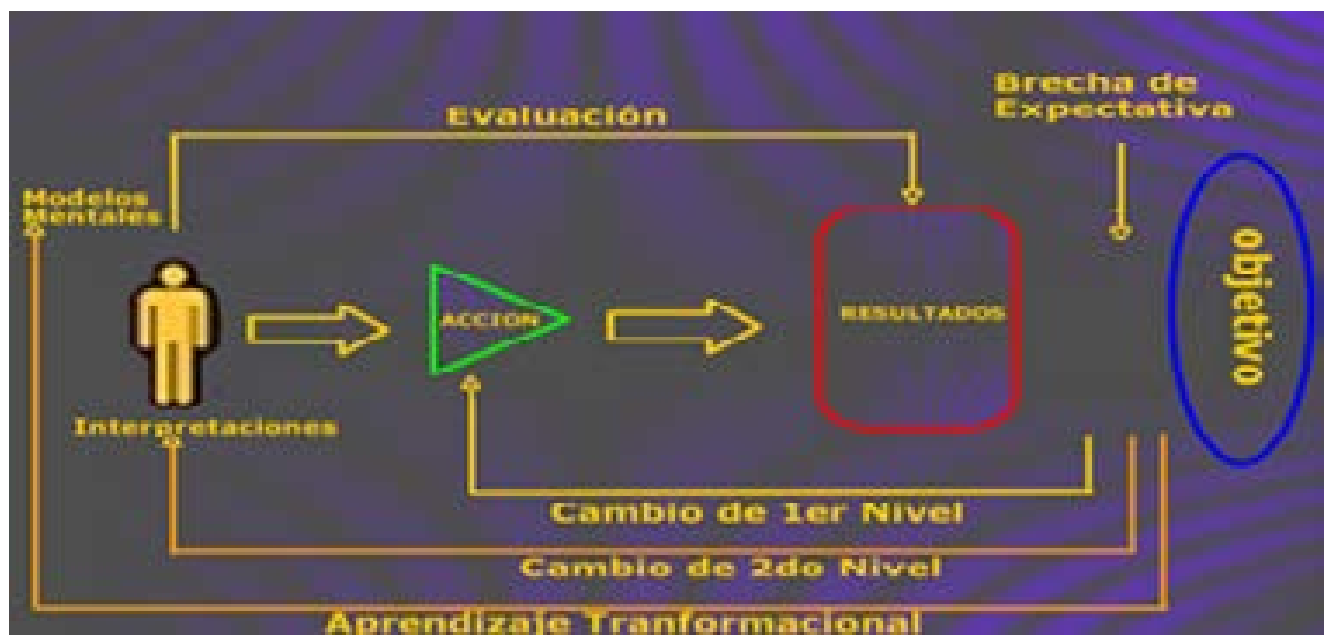

Fuente: Echeverría 2013

Esta concepción del modelo del observador, vista como un proceso de aprendizaje se transforma en instrumento generativo de oportunidades, clave para las instituciones públicas del Municipio Valledupar, por que logra presentar nuevas perspectivas para el docente y los estudiantes que entregan la posibilidad de modificar la realidad del aprendizaje del saber matemático logrando un cambio contundente en acto educativo.

\section{Etapas del aprendizaje matemático}

A partir de la teoría Genética, Piaget se plantean las características del aprendizaje, respetando el proceso de asimilación, acomodación adaptación y equilibrio, marcando un cambio cognitivo que tiene lugar cuando el esquema mental produce un caos para nuevamente establecer un equilibrio. En este sentido, las etapas del aprendizaje matemático, están inmersas en los procesos de aprendizaje, pues sus significados se construyen partiendo de las experiencias y conocimientos adquirido, permitiendo la estimulación del pensamiento lógico matemático vinculado a los conceptos o procesos matemáticos con otras áreas de contenido, e incluso en los aspectos de la vida cotidiana. (Inteligencia lógica de las matemáticas).

A continuación, se caracterizan las etapas del saber matemático por Sadovsky, (2004):

\section{- Introducción a la didáctica:}

La etapa de introducción como su nombre lo indica, está referida a la fase inicial, en la cual el docente hace una breve mención del tema a tratar durante la jornada. Existiendo múltiples formas de dar inicio a la clase de matemáticas, una de ellas es a través de la descripción breve del contenido a desarrollar, empleando la metodología de preguntas a los estudiantes cuyo objeto es abrir el debate y la reflexión sobre el tema; otras técnicas sugerida son el resumen del contenido de clases anteriores; el uso de historias, actualidad, información en internet, fenómenos naturales, juegos, entre otras. 
Dentro de uno de los objetivos del campo de la didáctica matemática, está el poder desarrollar en los estudiantes los procesos de pensamiento vinculados a contenidos de medición espacial, variación, y conteo (MEN, 2006), resaltando el pensamiento variacional y los sistemas algebraicos, que de una u otra forma están vinculadas con otras formas de pensamientos $y$, por ende, a los procesos inherentes a él mismo, convirtiéndose en factores relevantes al momento de dar respuesta a problemas puntuales, y al modelaje de situaciones reales de la vida misma. (MEN, 1998; MEN, 2006)

En este mismo orden de ideas, la transposición didáctica, el contrato didáctico y los campos conceptuales, tal como lo señala (Brousseau 2007), son soportes que validan el proceso de aprendizaje; ya que su aporte va, desde el poder transformar el conocimiento matemático para ser enseñado, pasando por las etapas del saber al saber enseñar, así como la transformación de las nociones sobre matemáticas al proceso de descripción de la misma, y la intrínseca economía que gira en torno a la organización del saber. Chevallard (1997).

Por otro lado, el contrato didáctico, es clave, pues se construyen en conjunto con las directrices que rigen el proceso de aprendizaje, que permite llegar al significado específico del conocimiento elaborado por los estudiantes; su importancia radica, en generar respuestas a un problema en función del proceso de decodificación de las convenciones didácticas implícitas, no necesariamente, según un razonamiento matemático lógico. Brousseau, (1983).
Desarrollo del saber matemático escolar. En esta fase, el docente de matemáticas asume la dirección y guía la participación de los estudiantes, ejecutando los nuevas secuencializaciones de los saberes matemáticos escolares, a través de preguntas y respuestas, bien sea con participación directa del estudiante, o por interrogantes abiertas al grupo para la resolución de situaciones problemas; de allí, que una de las estrategias más empleadas para la obtención de respuestas a problemas puntuales, pueden ser las preguntas elaboradas para inducir a un proceso de reflexión acerca de las distintas opciones para solucionar el problema planteado.

Cabe acotar, que el proceso del aprendizaje matemático involucra tanto al que aprende como al que asume el rol de enseñar; esta área específica, requiere disciplina en la planificación del material de estudios, reforzando conocimientos didácticos y de las áreas que se vinculan a los problemas incluso extra matemáticos; por ende, el docente debe ser un formador de seres humanos capaces de aprender a pensar con criterio propio, al evaluar alternativas para dar con la mejor opción a la solución del problema.

En función de ello, en el proceso de aprendizaje transformacional, se trabajan tanto saberes matemáticos escolares, tanto intra como extra matemáticos, orientando los objetivos hacia la apropiación del conocimiento comprobable, a través del dominio de los procedimientos matemáticos útiles para la resolución de las situaciones problemas. El énfasis del docente, es tratar de lograr que el alumno asimile la construcción de los algoritmos y aprenda a comprender el significado de ello. 
- Unidad de conocimiento. Esta etapa suele tener escasa frecuencia en los reportes, tales como el TIMSS y el PISA, sin embargo, a pesar de ello, está presente en forma implícita durante la ejecución de todas las otras etapas del aprendizaje matemático. En un contexto único, se dice que las matemáticas forman un mundo compuesto por un infinito de partículas vinculadas y entrelazadas unas entre sí, asemejándose a un árbol de múltiples ramificaciones, en función de ello, el docente busca conectar distintas ideas alrededor del problema para explicar el concepto tratado.

De allí que, la conectividad del saber matemático escolar, está estrechamente relacionado con la concepción de ideas básicas en educación matemática (Bruner, 1980; Mora, 2003; Schweiger, 1992) observándose como, por ejemplo, el concepto de triángulo permite ser trabajado desde varias aristas o puntos de vistas geométricos, así como también algunos saberes de trigonometría sobre los sólidos u otros conceptos matemáticos de mayor envergadura. En función de ello, los docentes de básica primaria requieren hacer explícitos los indicadores de los procesos generales de aprendizaje, los saberes matemáticos escolares y el contexto, durante el proceso de aprendizaje y enseñanza; por ello, es pertinente presentar esta fase de manera independiente, para conectar con diferentes conocimientos matemáticos en la resolución de situaciones problemas tanto externos como internos.

Profundización de conocimiento matemático y del lenguaje. Implica ir más allá de los conceptos, para ello es necesario conocer el proceso de aprendizaje, el interés del estudiante por adquirir el conocimiento, la práctica constante, las actividades académicas propuesta en cada sección de aula, y la normativa que regula el proceso de enseñanza-aprendizaje en cada una de las clases de matemáticas permeadas por dar un significado único a la comunicación, siempre en constante evolución; es decir, el paso de la matemática enseñada por parte del docente al estudiante, se da en una situación comunicativa por demás fuerte (D’Amore B. 2007). En virtud de ello, se puede interpretar que el aprendizaje de las matemáticas es un proceso que necesita intervenir en la interacción de los elementos actores en el proceso de enseñanza-aprendizaje y en su práctica docente profesional, disciplinar e interdisciplinar.

Con referencia a lo anterior, es importante resaltar que el ser humano promedio olvida lo aprendido con la ausencia de la práctica; es decir, es necesario implementar situaciones didáctica en contexto, que permitan consolidar el la construcción del conocimiento escolar; situación esta, que invita al docente a buscar apoyo en los conocimientos previos que tiene el estudiante, rescatando su experiencia y flexibilidad para llenar los vacíos o insuficiencia del dominio de los saberes matemáticos y del lenguaje escolares específicos, en función del aprendizaje matemático. Por consiguiente, la comprensión y la reflexión relacionadas con el aprendizaje del tercer orden, se vuelven en elementos claves para la consolidación del conocimiento.

- Inspección y evaluación del conocimiento. La inspección y evaluación, del conocimiento aportan al docente durante el proceso de aprendizaje información relativa, a la efectividad en los resultados de la enseñanza; con la aclaratoria, de que aún existen el control sobre los procesos, y la clasificación del estudiante en función de las normas preestablecidas del sistema. 
Cabe destacar, que la razón de ser del proceso de enseñanza es lograr el aprendizaje con la consolidación del conocimiento aprendido, evaluando a través de indicadores el nivel que el estudiante realmente alcanzó, en relación a la meta contenida en los planes de estudio; sin embargo, la didáctica en la enseñanza de la matemática es el camino a seguir, para establecer un mecanismo que permita medir a ciencia cierta el nivel del conocimiento real trabajado en las aulas de clase y fuera de ellas.

En este sentido, se recomienda utilizar distintos fundamentos metodológicos para inspeccionar el nivel del conocimiento matemático; como por ejemplo las peguntas preestablecidas, o las elaboradas durante y post del proceso de enseñanza, pues la respuesta dada por el estudiante permite la evaluación específica sobre el logro del aprendizaje; es decir, el proceso de verificación y validación tanto del cómo, así como del resultado, permiten canalizar el nivel de acompañamiento que necesita el estudiante para la construcción del saber matemático escolar y escolarizado en contexto.

Otra estrtegia recomendada es la observación individual del trabajo colectivo, pues permite al docente a través de la asignación de tareas de investigación, foros, debates y exposiciones, entre otras, verificar el comportamiento y desenvolvimiento del estudiante con base al proceso de aprendizaje, de esta manera disminuir las pruebas escritas que tanta angustia causa a los estudiantes.

- Corrección y alineación de errores. La construcción del dominio del conocimiento matemático escolar, en los últimos años se ha visto influenciado por la idea de una educación ajustada al formalismo, afectando desde la raíz a su razón de ser, reduciendo o minimizando el error como un elemento fundamental del proceso de aprendizaje de cualquier ciencia, especialmente del área matemática.

En contexto, la corrección y alineación de errores, es concebida como la oportunidad que tiene el docente para documentar las formas que no funcionan y construir elementos que sean aprovechados positivamente, sin penalizar ni causar frustración de allí que (Radatz, 1980), afirma que todos los seres humanos comenten cotidianamente errores; sin embargo, se requiere construir una cultura que tome el error como oportunidad de aprendizaje.

De allí que, Baruck (2004) señala en su obra "Qué edad tiene el capitán" la ingente necesidad existente de reordenar y encausar la postura del docente en función del error, señalando que el mismo esta intrínsecamente relacionado con el trabajo matemático escolar y son un motor que impulsa al estudiante a buscar respuestas, indagando sobre los conceptos y razonamientos lógicos matemáticos.

La reflexión de este proceso, encamina al docente hacia el concepto clave del aprendizaje transformacional, evaluando una manera distinta sobre el abordaje de los contenidos y situaciones problémica del saber matemático escolar, que apoyan al estudiante a interpretar con sentido y significado la experiencia de una ciencia aplicada en su entorno.

\section{B) Fundamentación Metodología}

La presente investigación está enmarcada bajo el enfoque empírico del paradigma positivista Martínez (2018), de tipo descriptiva interpretativa. 
Chávez (2008), ofrece la posibilidad de establecer y caracterizar, desde un punto de vista educativo, los procesos de aprendizaje en el saber matemático escolar en la educación básica primaria, de las Instituciones Públicas del Municipio de Valledupar. Su diseño, es no experimental transeccional de campo, (Sabino, 2007), ya que se recogen los datos obtenidos directamente de la experiencia Hernández, Fernández y Batista (2010).

Para efectos de la muestra, se considera la participación de cuarenta y seis (46) docentes; que según Tamayo y Tamayo (2005: 56), puede considerarse censal por ser finita y accesible, tal como lo muestra en la tabla No 1.

Tabla 1 Características de la muestra

\begin{tabular}{|l|l|l|l|l|l|}
\hline $\mathrm{N}$ & Instituciones & $\begin{array}{l}\text { No } \\
\text { Docentes }\end{array}$ & & Instituciones & $\begin{array}{l}\text { No } \\
\text { Docentes }\end{array}$ \\
\hline 1 & $\begin{array}{l}\text { Inst. Alfonso } \\
\text { López }\end{array}$ & 6 & 7 & $\begin{array}{l}\text { Inst. Loperena } \\
\text { Central }\end{array}$ & 6 \\
\hline 2 & $\begin{array}{l}\text { Inst. Raúl } \\
\text { Casimiro }\end{array}$ & 4 & 8 & $\begin{array}{l}\text { CASD. Simón } \\
\text { Bolívar }\end{array}$ & 4 \\
\hline 3 & $\begin{array}{l}\text { Inst. Consuelo } \\
\text { Araujo }\end{array}$ & 4 & 9 & $\begin{array}{l}\text { Inst. Pedro Castro } \\
\text { M }\end{array}$ & 4 \\
\hline 4 & Tec. José Eugenio & 4 & 1 & Tec. La Esperanza & 4 \\
\hline 5 & $\begin{array}{l}\text { Inst. Leonidas } \\
\text { Acuña }\end{array}$ & 2 & 1 & Tec. Upar & 4 \\
\hline 6 & Inst. Loperena G & 4 & $/$ & TOTAL & 46 \\
\hline
\end{tabular}

Fuente: Secretaria de Educación Municipal de Valledupar, Cesar (2015).

\section{C) Análisis de los resultados}

La tabla 2, procesos de aprendizaje permite deducir que el indicador con más alto promedio está constituido por el aprendizaje de primer orden, seguido del segundo orden, siendo el indicador más bajo el de tercer orden; esta realidad evidencia que los docentes se sienten más cómodos practicando el aprendizaje mecánico que diseñando y planificando estrategias, que les permitan relacionar directamente los procesos de aprendizaje en el saber matemático escolar con acciones efectivas.
Entonces, el docente tiene como reto aprender a aprender, tal como lo señala Echeverría (2007), el aprendizaje en el saber matemático escolar, implica apresurarse a la actitud de desaprender, (habilidad para soltar), haciendo necesario que los docentes dejen atrás los modelos mentales usados a través de años, que evidentemente están limitando el proceso. 
Tabla 2 Procesos del aprendizaje

\begin{tabular}{|c|c|c|c|c|c|c|c|}
\hline Indicador & Items & $\mathbf{S}$ & CS & AV & CN & $\mathbf{N}$ & $\begin{array}{l}\text { Promedio } \\
\text { Indicador }\end{array}$ \\
\hline \multirow{5}{*}{$\begin{array}{l}\text { Aprendizaje de } \\
\text { primer orden }\end{array}$} & $\begin{array}{l}\text { Utiliza preguntas verificando experiencias que motiven al } \\
\text { desarrollo de un pensamiento independiente }\end{array}$ & $33 \%$ & $39 \%$ & $17 \%$ & $11 \%$ & $0 \%$ & \multirow{5}{*}{3,69} \\
\hline & $\begin{array}{l}\text { Incorpora elementos novedosos en las clases enriqueciendo el } \\
\text { aprendizaje }\end{array}$ & $13 \%$ & $39 \%$ & $37 \%$ & $11 \%$ & $0 \%$ & \\
\hline & $\begin{array}{l}\text { Toma consciencia que cuando cambia las perspectivas de la } \\
\text { enseñanza se modifican las interpretación de sus estudiantes }\end{array}$ & $13 \%$ & $37 \%$ & $46 \%$ & $4 \%$ & $0 \%$ & \\
\hline & Promedios & $20 \%$ & $38 \%$ & $33 \%$ & $9 \%$ & $0 \%$ & \\
\hline & Resultados & \multicolumn{3}{|c|}{$91 \%$} & \multicolumn{2}{|c|}{$9 \%$} & \\
\hline \multirow{5}{*}{$\begin{array}{l}\text { Aprendizaje de } \\
\text { segundo orden }\end{array}$} & $\begin{array}{l}\text { Incorpora nuevas acciones en la ejecución de las tareas } \\
\text { considerando otras perspectiva de la situación problema }\end{array}$ & $4 \%$ & $13 \%$ & $70 \%$ & $11 \%$ & $2 \%$ & \multirow{5}{*}{3,41} \\
\hline & Considera que la responsabilidad es de los estudiantes. & $52 \%$ & $24 \%$ & $7 \%$ & $17 \%$ & $0 \%$ & \\
\hline & Considera una solución única frente a la situación problema & $11 \%$ & $4 \%$ & $65 \%$ & $20 \%$ & $0 \%$ & \\
\hline & Promedios & $22 \%$ & $14 \%$ & $47 \%$ & $16 \%$ & $1 \%$ & \\
\hline & Resultados & & $33 \%$ & & & & \\
\hline \multirow{5}{*}{$\begin{array}{l}\text { Aprendizaje de } \\
\text { tercer orden, } \\
\text { transformacional }\end{array}$} & $\begin{array}{l}\text { Fomenta ejercicios prácticos para producir cambios de } \\
\text { conducta en los estudiantes. }\end{array}$ & $15 \%$ & $9 \%$ & $43 \%$ & $28 \%$ & $4 \%$ & \multirow{5}{*}{3,12} \\
\hline & $\begin{array}{l}\text { Considera que el desvio de la atención de los estudiantes es } \\
\text { producto del desinteres académico. }\end{array}$ & $39 \%$ & $37 \%$ & $2 \%$ & $20 \%$ & $2 \%$ & \\
\hline & Sincronizando la practica con la reflexión en el aula. & $4 \%$ & $0 \%$ & $41 \%$ & $43 \%$ & $11 \%$ & \\
\hline & Promedios & $20 \%$ & $15 \%$ & $29 \%$ & $30 \%$ & $6 \%$ & \\
\hline & Resultados & \multicolumn{2}{|c|}{$35 \%$} & \multicolumn{3}{|c|}{$65 \%$} & \\
\hline
\end{tabular}

Fuente: Elaboración propia.

\section{ETAPAS DEL APRENDIZAJE EN EL SABER MATEMÁTICO ESCOLAR.}

Los resultados reflejados en la tabla 3 , etapas del aprendizaje del saber matemático escolar, permiten evidenciar que, el indicador con el promedio más bajo esta reflejado en el desarrollo del contenido; el de mayor promedio se muestra en la consolidación de conocimiento, demostrando que ciertamente los docentes dentro de su ejecución contemplan contenidos relacionados con la cotidianidad y las necesidades presentadas por el estudiante, seguido por la corrección y alineación de errores, con un moderado dominio, denotando una postura rígida de los docentes al no considerar el error, como una fuente de información que ofrece elementos y datos relevantes para fortalecer el proceso de aprendizaje de los estudiantes en el saber matemático.

En tal sentido, autores como (Nesher, 2000; Blum, 1985; Mora, 2002) señalan que el proceso de consolidación del aprendizaje de las matemáticas escolares, su norte es estar estrechamente vinculado a la calidad del contenido que se imparte en el salón de clases, así como las estrategias de enseñanza que emplea el docente $y$, sobre todo, la relación entre matemática y disciplina. 
Tabla 3 Etapas del aprendizaje

\begin{tabular}{|c|c|c|c|c|c|c|c|}
\hline Indicador & Items & $\mathbf{S}$ & CS & AV & CN & $\mathbf{N}$ & $\begin{array}{l}\text { Promedio } \\
\text { Indicador }\end{array}$ \\
\hline \multirow{5}{*}{$\begin{array}{l}\text { Introducción a la } \\
\text { didáctica }\end{array}$} & $\begin{array}{l}\text { Considera los temas trabajados con anterioridad en el comienzo } \\
\text { de las actividades diarias }\end{array}$ & $37 \%$ & $28 \%$ & $13 \%$ & $15 \%$ & $7 \%$ & \multirow{5}{*}{3,62} \\
\hline & $\begin{array}{l}\text { Plantea preguntas que le permitan a los estudiantes familiarizarse } \\
\text { con el tema que se abordará. }\end{array}$ & $13 \%$ & $26 \%$ & $41 \%$ & $17 \%$ & $2 \%$ & \\
\hline & $\begin{array}{l}\text { Vincula el lenguaje natural con la manipulación de objetos } \\
\text { concretos activando la atención plena de los estudiantes }\end{array}$ & $22 \%$ & $46 \%$ & $28 \%$ & $2 \%$ & $2 \%$ & \\
\hline & Promedios & $24 \%$ & $33 \%$ & $28 \%$ & $12 \%$ & $4 \%$ & \\
\hline & Resultados & \multicolumn{3}{|c|}{$85 \%$} & \multicolumn{2}{|c|}{$15 \%$} & \\
\hline \multirow{5}{*}{$\begin{array}{l}\text { Desarrollo del } \\
\text { contenido }\end{array}$} & $\begin{array}{l}\text { Incorpora en su discurso nuevos términos matemáticos que } \\
\text { generan conflicto en los estudiantes }\end{array}$ & $0 \%$ & $0 \%$ & $13 \%$ & $17 \%$ & $70 \%$ & \multirow{5}{*}{2,51} \\
\hline & $\begin{array}{l}\text { Utiliza más de una situación problémica generando confusión en } \\
\text { los estudiantes. }\end{array}$ & $0 \%$ & $7 \%$ & $15 \%$ & $50 \%$ & $28 \%$ & \\
\hline & $\begin{array}{l}\text { Formula proposiciones sencillas orientando al estudiante hacia } \\
\text { nuevos procedimientos que lleven a la solución del problema }\end{array}$ & $30 \%$ & $52 \%$ & $13 \%$ & $4 \%$ & $0 \%$ & \\
\hline & Promedios & $10 \%$ & $20 \%$ & $14 \%$ & $24 \%$ & $33 \%$ & \\
\hline & Resultados & \multicolumn{2}{|c|}{$30 \%$} & \multicolumn{3}{|c|}{$70 \%$} & \\
\hline \multirow{5}{*}{$\begin{array}{l}\text { Unidad de } \\
\text { conocimiento }\end{array}$} & $\begin{array}{l}\text { 25. Utiliza en su discurso diferentes ideas construyendo un } \\
\text { determinado concepto matemático }\end{array}$ & $17 \%$ & $15 \%$ & $57 \%$ & $9 \%$ & $2 \%$ & \multirow{5}{*}{3,35} \\
\hline & $\begin{array}{l}\text { 26. Aumenta el grado de complejidad del conocimiento } \\
\text { considerando el nivel de aprendizaje del grupo de estudiantes. }\end{array}$ & $13 \%$ & $37 \%$ & $30 \%$ & $20 \%$ & $0 \%$ & \\
\hline & $\begin{array}{l}\text { Conecta con diferentes formas del saber aclarando dudas sobre } \\
\text { el contenido abordado en clase }\end{array}$ & $11 \%$ & $13 \%$ & $65 \%$ & $11 \%$ & $0 \%$ & \\
\hline & Promedios & $14 \%$ & $22 \%$ & $51 \%$ & $13 \%$ & $1 \%$ & \\
\hline & Resultados & & $86 \%$ & & & & \\
\hline \multirow{5}{*}{$\begin{array}{l}\text { Consolidación del } \\
\text { conocimiento }\end{array}$} & $\begin{array}{l}\text { Plantea ejercicios relacionados con la cotidianidad que } \\
\text { mantengan presentes los contenidos aprendidos }\end{array}$ & $63 \%$ & $15 \%$ & $13 \%$ & $7 \%$ & $2 \%$ & \multirow{5}{*}{3,64} \\
\hline & $\begin{array}{l}\text { Evalúa la calidad de los ejercicios sin considerar la participación } \\
\text { de los estudiantes }\end{array}$ & $13 \%$ & $20 \%$ & $20 \%$ & $46 \%$ & $2 \%$ & \\
\hline & $\begin{array}{l}\text { Vincula las actividades diarias con las necesidades presentes en } \\
\text { los estudiantes }\end{array}$ & $17 \%$ & $46 \%$ & $24 \%$ & $11 \%$ & $2 \%$ & \\
\hline & Promedios & $31 \%$ & $27 \%$ & $19 \%$ & $21 \%$ & $2 \%$ & \\
\hline & Resultados & & $77 \%$ & & & & \\
\hline \multirow{5}{*}{$\begin{array}{l}\text { Profundización del } \\
\text { conocimiento }\end{array}$} & $\begin{array}{l}\text { Profundiza los contenidos matemáticos respetando el desarrollo } \\
\text { cognitivo de los estudiantes }\end{array}$ & $41 \%$ & $15 \%$ & $24 \%$ & $17 \%$ & $2 \%$ & \multirow{5}{*}{3,55} \\
\hline & $\begin{array}{l}\text { Selecciona temas interesantes que despierten la motivación de } \\
\text { los estudiantes. }\end{array}$ & $22 \%$ & $39 \%$ & $13 \%$ & $24 \%$ & $2 \%$ & \\
\hline & $\begin{array}{l}\text { Utiliza estrategias concretas en las operaciones matemáticas } \\
\text { comprobando el efecto en el aprendizaje }\end{array}$ & $17 \%$ & $30 \%$ & $28 \%$ & $17 \%$ & $7 \%$ & \\
\hline & Promedios & $27 \%$ & $28 \%$ & $22 \%$ & $20 \%$ & $4 \%$ & \\
\hline & Resultados & & $77 \%$ & & & & \\
\hline \multirow{5}{*}{$\begin{array}{l}\text { Inspección y } \\
\text { evaluación del nuevo } \\
\text { conocimiento }\end{array}$} & 34. Aplica evaluaciones largas para verificar el aprendizaje & $2 \%$ & $0 \%$ & $28 \%$ & $43 \%$ & $26 \%$ & \multirow{5}{*}{3,38} \\
\hline & $\begin{array}{l}\text { 35. Mezcla situaciones reales vividas en clase verificando el } \\
\text { aprendizaje }\end{array}$ & $48 \%$ & $26 \%$ & $13 \%$ & $11 \%$ & $2 \%$ & \\
\hline & 36. Redacta sus evaluaciones considerando un patron constante. & $30 \%$ & $43 \%$ & $22 \%$ & $4 \%$ & $0 \%$ & \\
\hline & Promedios & $27 \%$ & $23 \%$ & $21 \%$ & $20 \%$ & $9 \%$ & \\
\hline & Resultados & & $71 \%$ & & & & \\
\hline \multirow{5}{*}{$\begin{array}{l}\text { Corrección y } \\
\text { alineación de errores }\end{array}$} & $\begin{array}{l}\text { Conserva el formalismo de las evaluaciones con una estructura } \\
\text { definida. }\end{array}$ & $33 \%$ & $39 \%$ & $20 \%$ & $4 \%$ & $4 \%$ & \multirow{5}{*}{3,12} \\
\hline & Considera el error como parte del proceso de aprendizaje & $9 \%$ & $11 \%$ & $4 \%$ & $67 \%$ & $9 \%$ & \\
\hline & $\begin{array}{l}\text { Utiliza sus errores mejorando su lenguaje en el desempeño } \\
\text { docente }\end{array}$ & $11 \%$ & $9 \%$ & $54 \%$ & $24 \%$ & $2 \%$ & \\
\hline & Promedios & $17 \%$ & $20 \%$ & $26 \%$ & $32 \%$ & $5 \%$ & \\
\hline & Resultados & \multicolumn{2}{|c|}{$37 \%$} & \multicolumn{3}{|c|}{$63 \%$} & \\
\hline
\end{tabular}

Fuente: Elaboración propia. 


\section{CONCLUSIONES Y RECOMENDACIONES.}

Según los resultados obtenidos, el aprendizaje del primer orden presenta un alto dominio, ubicándose por encima del segundo y del tercero, lo cual evidencia que los docentes continúan arraigados en esquemas convencionales; por ende, se muestra gran dificultad a la hora de cohesionarlas con las etapas del saber matemático y con acciones que involucren el área emocional del estudiante.

Por ello se recomienda, incorporar recursos innovadores que conecte al estudiante con conocimientos relevantes, facilitando la resolución de situaciones problemas, correlacionando los objetivos con las secuencias de saberes matemáticos y del lenguaje escolares y las necesidades del contexto, que sirvan para dar valor a su propio significado.

Ahora bien, para la caracterización las etapas del saber matemático escolar, se encuentra que el indicador con más resaltante es la consolidación del conocimiento, reflejando que la dificultad que tienen los docentes para que el estudiante consolide lo aprendido y lo ponga en práctica, en vista de los anterior se recomienda la apropiación de su saber especifico de manera significativa y sostenida para poderlo llevar al estudiante de forma sencilla; impactando el quehacer pedagógico, en beneficio de todo el sistema.

En este orden, respondiendo a la pregunta problema ¿cómo el proceso de aprendizaje influye en el saber matemático escolar?, se deduce según los resultados arrojados que, cuando el docente hace consciente que el proceso del aprendizaje, se conforma desde la biología del ser humano, pueden realizar cambios pertinentes en su quehacer pedagógico, así como también reconocer cual es el nivel de aprendizaje del estudiante, para poder coordinar las etapas del saber matemático escolar atendiendo a las características particulares de los mismos tomando el rol de líder hacia el significado del saber especifico.

\section{REFERENCIAS BIBLIOGRÁFICAS}

Baruck, Y (2004). Managing careers: Theory and practice. Harlow, UK: FT-Prentice Hall/ Pearson

Brousseau, G. (2007). Iniciación al estudio de la teoría de las situaciones didácticas. Buenos Aires: Libros del Zorzal.

Chávez, N. (2008). Introducción a la Investigación Educativa. Ediciones Astrodata. C.C. Maracaibo - Venezuela.

Chevallard, Y. (1997). La Transposición Didáctica: del saber sabio al saber enseñado. Buenos Aires. Segunda edición.

Chiva R. (2012). Aprendizaje organizativo y los sistemas complejos. Universidad Jaume. Publicaciones de la Universidad de Jaume

Echeverría (2007). La empresa Emergente. Buenos Aires. Ediciones Granica.

Echeverría R. (2013), Escritos sobre Aprendizaje. Comunicaciones Nor-Este. Santiago de Chile.

Hernández, R., Fernández, C., y Baptista, P. (2010). Metodología de la Investigación. México D.F.: Mcgraw-HILL / Interamericana Editores, S.A. de C.V 
Martínez R. (2018), Metodología de la investigación. Editorial CEGAGE. Learning. Ciudad de México

Mier, H. (2004). El Planteamiento Estratégico de las Organizaciones Públicas. Ediciones Granica Buenos Aires

Ministerio de Educación Nacional [MEN]. (2012). El perfil del docente orientador que se rige por el Estatuto de Profesionalización Docente (Decreto Ley 1278 de 2002). Bogotá: MEN

Mora, M (2002). La teoría de las representaciones sociales de Serge Moscovici. Athenea Digital

Muniz y Borges, (2008). Utilización del Lenguaje Matemático como instrumento para reflexión sobre la enseñanza aprendizaje: caso de redacción en las Matemáticas. Campiñas: Segundo Seminario de Historias en las Aulas de Matemática (SHIAM).

Nesher, P. (2000). Posibles relaciones entre lenguaje natural y lenguaje matemático en Matemáticas y educación. Retos y cambios desde una perspectiva internacional. España: Graó.

Polya, G. (1989). Fases para resolver un problema. Obtenido de https://www.ecured. cu/Resoluci\%C3\%B3n_de_Problemas Matem\%C3\%A1ticos
Radatz, H. (1980). Students Errors in the matematiccs LearningProcess: a Survey for the Learning of mathematics. Vol.1

Roegiers X. (2004). L'École et l'Évaluation: Des Situations pour Évaluer les Compétences des Élèves. Bruxelles: De Boeck.

Sabino, C. (2007). El proceso de investigación. Editorial Panapo. 2da reimpresión. Caracas Colombia.

Solaz, López y Gómez (2011), Aprendizaje basado en problemas en la Educación Superior: una metodología necesaria en la formación del profesorado. Editores Didáctica De Las Ciencias Experimentales y Sociales. N. ${ }^{\circ}$ 25. 2011, 177-186 (ISSN 0214-4379).

Tamayo y Tamayo (2005). El proyecto de investigacion. Instituto Colombiano para el Fomento de la Educación Superior, ICFES

Vygotsky, L. (1988). El desarrollo de los procesos psicológicos superiores. México: Grijalbo

Wolk, L. (2007). El arte de soplar brasas en acción. Gran Aldea Editores.

Woolfolk, A. (2006). Psicología Educativa. México: Pearson Educación. 